\title{
nature
}

nanotechnology

\section{Physics in the nanoworld}

\author{
From nanomechanical mass sensors to superconducting nanobolometers for astrophysics, \\ the links between physics and nanoscience and technology are deep and varied.
}

The Large Hadron Collider (LHC) due to start taking data at the CERN particle physics laboratory in Geneva this summer - has every right to call itself the biggest experiment in the world. However, the purpose of this colossal undertaking, which involves accelerating proton beams to energies of $7 \mathrm{TeV}$ in an underground tunnel with a circumference of $27 \mathrm{~km}$ and then analysing the debris produced in collisions between the protons, is to explore the nature of matter at length scales many orders of magnitude shorter than the nanoscale ${ }^{1}$. Once upon a time particle physicists referred to their colliders somewhat prosaically as microscopes, but the LHC is being marketed as a big-bang machine that could find evidence for the Higgs boson (and hence reveal the origins of mass), and possibly more exotic phenomena such as supersymmetry or extra dimensions that would tell us more about the fundamental nature of space and time.

Of course, going to great lengths to learn about the behaviour of matter on shorter and shorter length scales is familiar to nanoscientists as well as particle physicists. Conventional microscopists had to build taller machines to reach the higher energies and the shorter de Broglie wavelengths needed to study samples in finer detail, and, again like their particle physics brethren, they had to develop ever-more complex beamfocusing optics to get the most from their experiments. Indeed, physicists laid much of the groundwork for what we now call nanoscience and technology, so it is not surprising that they play such a prominent role in the nanoworld.

The scanning tunnelling microscope and the atomic force microscope offered a new way of exploring matter at the level of atoms and molecules ${ }^{2}$. Instead of exploiting the wave-like nature of particles and all the challenges this entails, scanning probe devices operated in real space and actually measured something — such as a tunnelling current or a force - as a sharp tip on a flexible cantilever was scanned over the surface in question. A similar direct approach is taken by physicists working with nanoelectromechanical devices, such as the nanomechanical mass sensor developed by Kenneth Jensen, Kwanpyo Kim and Alex Zettl ${ }^{3}$. By monitoring how the vibration frequency of a double-walled carbon nanotube clamped at one end changes as gold atoms become attached to it, Jensen and co-workers demonstrate atomic resolution with their device.

\section{It is not surprising that physicists} play such a prominent role in the nanoworld.

Scanning probe microscopy left the physics laboratory many years ago, but researchers in various fields of nanoscience and technology are still busy improving its capabilities and applying it in new ways. The latest example is the use of scanning near-field ultrasonic holography to probe inside cells, as described by Ali Passian and co-workers on page 501 . The ability to detect nanoparticles below the cell surface could make this technique useful for studying the toxicity of nanomaterials.

Back in the physics lab, meanwhile, new types of devices continue to take shape. On page 482, for instance, Daniel Koenig and co-workers describe a nanomechanical single-electron shuttle that is driven by ultrasound waves. In the past, such devices have been driven electrically, leading to unwanted interactions between the device and its drive mechanism. This new approach could lead to the development of highperformance nanomechanical singleelectron devices for applications such as metrology, and possibly a new standard for the ampere.

Graphene continues to excite physicists and many others ${ }^{4}$. In addition to being a rich source of new physics, this two-dimensional form of carbon could have applications in electronics, but the novel electronic properties of graphene can be compromised when it is supported on an insulating substrate. However, as reported by Eva Andrei and co-workers on page 491 , and also by researchers at Columbia University ${ }^{5}$, suspended graphene samples can display lowtemperature mobility values much higher than those observed in semiconductors or non-suspended graphene. Real-world electronic devices based on graphene will also involve the use of metal contacts, and on page 486 Eduardo Lee and colleagues report how scanning photocurrent microscopy has revealed that these contacts lead to potential steps that act as transport barriers in graphene devices.

It is clear that most nanophysicists have their feet planted firmly on the ground, but others are looking to the stars. Michael Gershenson, Boris Karasik and co-workers, for example, have developed a superconducting hot-electron nanobolometer that might one day be used to detect far-infrared photons from the furthest reaches of the universe on a space-based telescope (page 496). It's all a long way from there being plenty of room at the bottom.

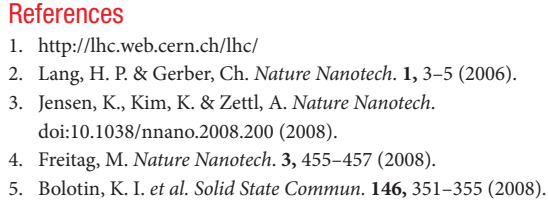

